\title{
LETTERS
}

\section{The relationship between sarcoidosis and lymphoma}

\section{To the Editors:}

Lymphocyte activation in sarcoidosis and the involvement of the reticuloendothelial system (liver, spleen, lymph nodes) make differential diagnosis between sarcoidosis and lymphoma a difficult task. In an earlier epidemiological study, an 11-fold increase in lymphoma cases among patients with sarcoidosis was observed; however, the methodology of this study has been questioned [1-3]. In order to elucidate the clinical characteristics of sarcoidosis and lymphoma, we conducted this retrospective review of cases of patients in which sarcoidosis and lymphoma coexisted in the same patient. These cases have been retrieved from our institution (University of Southern California, Los Angeles, CA, USA) and from an extensive Medline review.

Our inclusion criteria were: 1) biopsy-proven sarcoidosis, based on American Thoracic Society (ATS)/European Respiratory Society (ERS)/World Association of Sarcoidosis and Other Granulomatous Diseases (WASOG) criteria [4] applied by the authors retrospectively; 2) biopsy-proven lymphoma classified as Hodgkin Lymphoma (HL) and non-HL (NHL) according to the World Health Organization (WHO) classification [5]. Exclusion criteria were diagnosis of other malignancies and sarcoid-like reactions (SLR). These reactions, secondary to malignancies, are considered to occur either adjacent to the primary malignant site or to local drainage nodes. Group A consisted of cases in which sarcoidosis preceded lymphoma; group B consisted of cases in which the two diseases presented concurrently (in a period $<1$ month); and, in group C, lymphoma preceded sarcoidosis.

The primary end-point was to clarify the symptoms and signs 1) of lymphoma in a patient already diagnosed with sarcoidosis and 2) of sarcoidosis in a patient already diagnosed with lymphoma. We compared initial sarcoidosis presentation (group A) with initial lymphoma presentation (group C); then, sarcoidosis as the second disease (group C) with lymphoma as the second disease (group A). Our secondary end-point was to describe the diagnostic modalities most frequently proven effective for diagnosis. Data are presented as mean (95\% CI). Differences in measured values were calculated using the MannWhitney test. Fisher's exact test was used to compare qualitative characteristics. Statistics were calculated using GraphPad Prism 5 software (GraphPad Software, La Jolla, CA, USA).

112 cases were initially recognised. Three cases were attributed to mycobacterial infection, two were drug-induced, three cases had lymphomatoid granulomatosis and other nine had solidorgan malignanies. 14 cases exhibited SLRs, one had a granulomatous angiitis, and one did not fulfill the ATS criteria. In the majority of the remaining 79 cases, sarcoidosis preceded lymphoma (group A, $n=55,69.6 \%$ ). NHL was more common and the interval period between two diseases was significantly longer in group A. The distribution of lymphoma types and sarcoidosis, in stages through the three groups, was homogeneous. Subjects in group $\mathrm{C}$ had fewer organs involved in sarcoidosis compared with group A (table 1).

As preceding disease, common features of sarcoidosis were bilateral hilar lymphadenopathy (BHL) and parenchymal lung disease; whereas for lymphoma, splenomegaly and bone marrow involvement. Due to the small sample size statistical analysis was not performed. As a second diagnosis, significant differentiating features for sarcoidosis were BHL and lung disease, elevated angiotensin converting enzyme (ACE) levels and dyspnoea $(\mathrm{p}<0.05)$. Cough and skin involvement were marginally significant $(p=0.09)$. Cardinal features of lymphoma, when presented as second disease, were splenomegaly and biopsy-positive peripheral lymph nodes $(\mathrm{p}<0.05)$. Lung, skin, liver and mediastinum served as biopsy sites for sarcoidosis; spleen, bone marrow and laparotomy for lymphoma; and lymph nodes stations for both. Of the NHL cases, 14 were indolent and responded to treatment, while 12 were aggressive. 14 patients died shortly after diagnosis, nine due to NHL and five due to HL.

Sarcoidosis preceded lymphoma in most cases and NHL was more common than HL. In a sarcoidosis patient, new onset of lymphoma was diagnosed by splenic and new lymph node involvement. Conversely, in a patient with lymphoma, sarcoidosis was diagnosed on the basis of new respiratory symptoms, elevated ACE levels, BHL, pulmonary and, possibly, skin disease. Both sarcoidosis and lymphoma occurred frequently with constitutional symptoms, haematological abnormalities and similar organ involvement (mediastinum, liver and bone marrow). Consequently, diagnostic procedures performed were similar for the two diseases (with the exception of the pulmonary site for sarcoidosis and abdominal sites for lymphoma). When following disease, sarcoidosis occurred after a shorter period of about 3 yrs, and fewer organs were involved.

This letter is the first study to report on all sarcoidosislymphoma cases described in the literature. Since sarcoidosis may clearly coexist with haematologic malignancies, the latter posing a diagnostic challenge for clinicians, the aforementioned features could be of use in clinical practice and in the differential diagnosis procedure. Moreover, this study shows that sarcoidosis and lymphoma share many common features and that the pursuit of histological proof is warranted in every lymphoma, and almost every sarcoidosis, case.

The limitations of our study are publication biases, the retrospective application of ATS criteria, the masquerading nature of sarcoidosis itself, and the lack of a control population. Inevitably, the authors' criteria were used to classify reported cases as true sarcoidosis or as mimicking sarcoidosis, since tissue is not selfproving of sarcoidosis. However, the rarity of this coexistence 


\begin{tabular}{|c|c|c|c|c|c|}
\hline & All subjects & Group A & Group B & Group C & p-value \\
\hline Females & $40 / 77(52)$ & 29 & 3 & 8 & $>0.1$ \\
\hline Age at diagnosis with sarcoidosis yrs & $44(41-47)$ & $42(38-46)$ & $52(43-62)$ & $49(42-56)$ & $>0.1$ \\
\hline Interval between diseases months & & $96(69-123)$ & & $36(7-65)$ & $<0.05$ \\
\hline NHL & 48/76 (63) & 32 & 5 & 11 & $>0.1$ \\
\hline HL & 28/76 (37) & 20 & 0 & 8 & $>0.1$ \\
\hline Stage 0 & $11 / 73(15)$ & 8 & 1 & 2 & $>0.1$ \\
\hline Stage I & 21/73 (29) & 14 & 2 & 5 & $>0.1$ \\
\hline Stage II & $21 / 73(29)$ & 16 & 0 & 5 & $>0.1$ \\
\hline Dyspnoea & & $4 / 40$ & & $6 / 16$ & $<0.05$ \\
\hline Elevated ACE & & $0 / 40$ & & $4 / 16$ & $<0.05$ \\
\hline Lung & & $0 / 40$ & & $9 / 16$ & $<0.05$ \\
\hline BHL & & $2 / 40$ & & $8 / 16$ & $<0.05$ \\
\hline Skin & & $3 / 40$ & & $4 / 16$ & 0.09 \\
\hline Lymph nodes & & $20 / 40$ & & $0 / 16$ & $<0.05$ \\
\hline Spleen & & $13 / 40$ & & $0 / 16$ & $<0.05$ \\
\hline
\end{tabular}

Data are presented as $\mathrm{n} / \mathrm{N}(\%)$, mean $(95 \% \mathrm{Cl})$ or $\mathrm{n} / \mathrm{N}$, unless otherwise stated. NHL: non-Hodgkin Lymphomal; HL: Hodgkin Lymphoma; ACE: angiotensin-converting enzyme; BHL: bilateral hilar lymphadenopathy.

makes this approach reasonable. The end-point was to compare features of the two groups (A and $\mathrm{C}$ ), rather than comparing with a separate control population.

Our data are in agreement with the high positive predictive value of BHL for sarcoidosis versus lymphoma. This is particularly important, as lymphoma is also considered at the differential diagnosis of BHL. Lung disease and BHL are reported to occur in $>95 \%$ of sarcoidosis patients. Lung disease, as shown in our study, is uncommon in lymphomas. In HL, primary lung lymphoma is rare, whereas in NHL represents 5\% of extranodal sites [6]. Mediastinal lymph node enlargement, as well as constitutional B symptoms occurred in both sarcoidosis and lymphoma and were not helpful in differentiating between the two diseases.

Interestingly, patients in group C, post-lymphoma and its treatment, developed an active sarcoidosis mainly confined into the chest, as shown by the high ACE levels, the mean of two- organ disease, and by the observation that in nine out of 16 cases, lung was one of the two organs affected. While we may not preclude that distant SLRs occurred in group C, it is currently unknown how a malignant immunosuppressive effect or immune reconstitution after treatment may affect sarcoidosis. In the remaining seven cases, chemotherapy might have had an effect. However, drug-induced granulomatous lung disease has been reported only for bleomycin, as a component of the ABVD regimen (doxorubicin, bleomycin, vinblastine and dacarbazine) used against $\mathrm{HL}$, and only rarely (in two case reports).
Development of new lymph node disease and splenic involvement during the course of chronic sarcoidosis should be investigated promptly in order to rule out concomitant lymphoma. These findings correlate with the predominance of NHL in these selected cases. Nodal disease is common in NHL ( $>60 \%$ of cases). Bone marrow and spleen are affected in 30 $50 \%$ of patients, whereas spleen is affected in $\sim 7 \%$ of sarcoidosis cases. Asymptomatic splenomegaly occurs more often. Massive splenomegaly is rare and warrants, as shown also in our cases, exclusion of the malignancy [7].

Excisional biopsy of enlarged lymph nodes should be performed first. If not diagnostic, bone marrow biopsy established lymphoma in $15 \%$ of the cases and may be of help.Mediastinoscopy is diagnostic if chest disease is present. Splenectomy and laparotomy should be regarded late in the diagnostic assessment as well as a therapeutic procedure. Endobronchial ultrasound guided- transbronchial needle aspiration provides an alternative to mediastinoscopy, as it has been shown to be useful in diagnosing sarcoidosis in $88 \%$ of cases and lymphoma in $81-91 \%$ of cases, with a negative predictive value for lymphoma of $92.9 \%$ [8].

Studies based on cross-registry data have had inconsistent results regarding the prevalence of lymphomas in sarcoidosis patients. A recent Scandinavian study found a high risk for NHL, persistent 4 yrs after diagnosis of sarcoidosis [9]. This risk was associated with older age and multiple hospitalisations. Another cohort study, though, found no such risk in a population consisting mostly of African Americans with sarcoidosis [10]. 
Sarcoidosis and lymphoma, mainly NHL, may occur together, with sarcoidosis usually preceding lymphoma. Because many features of sarcoidosis and lymphoma are similar, histological confirmation of malignancy is necessary, especially if new nodal disease and splenomegaly are present. Sarcoidosis, although rare, may complicate the course of lymphoma. Prominent features of sarcoidosis were respiratory symptoms, high ACE levels, BHL in asymptomatic subjects and lung disease. Despite the limitations of this study, the above described features should alert clinicians to pursue a second diagnosis, in order either to properly diagnose malignancy in the sarcoidosis setting or to save cancer patient from a potentially toxic treatment escalation due to a presumed lymphoma recurrence.

\section{I.C. Papanikolaou* and O.P. Sharma ${ }^{\#}$}

*Third Pulmonary Dept, Sismanoglio General Hospital, Athens, Greece. "Pulmonary and Critical Care Divison, Keck School of Medicine, University of Southern California, Los Angeles, CA, USA.

Correspondence: I.C. Papanikolaou, Room 11-900, LAC-USC Medical Center, 1200 North State Street, Los Angeles, CA 90033, USA. E-mail: hliaspapa@hotmail.com

\section{REFERENCES}

1 Brincker $\mathrm{H}$, Wilbek E. The incidence of malignant tumours in patients with respiratory sarcoidosis. Br J Cancer 1974; 29: 247-251.

2 Rømer FK, Hommelgaard P, Schou G. Sarcoidosis and cancer revisited: a long-term follow-up study of 555 Danish sarcoidosis patients. Eur Respir J 1998; 12: 906-912.

3 Brincker $\mathrm{H}$. The sarcoidosis-lymphoma syndrome. $\mathrm{Br}$ J Cancer 1986; 54: 467-473.

4 Hunninghake GW, Costabel U, Ando M, et al. ATS/ERS/WASOG statement on sarcoidosis. Sarcoidosis Vasc Diffuse Lung Dis 1999; 16: 149-173.

5 Harris NL, Jaffe ES, Diebold J, et al. World Health Organization classification of neoplastic diseases of the hematopoietic and lymphoid tissues: Report of the Clinical Advisory Committee meeting-Airlie House, Virginia, November 1997. J Clin Oncol 1999; 17: 3835-3849.

6 Kim JH, Lee SH, Park J, et al. Primary pulmonary non-Hodgkin's lymphoma. Jpn J Clin Oncol 2004; 34: 510-514.

7 O'Reilly RA. Splenomegaly in 2,505 patients at a large university medical center from 1913 to 1995.1963 to 1995: 449 patients. West J Med 1998; 169: 88-97.

8 Cetinkaya E, Yildiz P, Altin S, et al. Diagnostic value of transbronchial needle aspiration by Wang 22-gauge cytology needle in intrathoracic lymphadenopathy. Chest 2004; 125: 527-531.

9 Askling J, Grunewald J, Eklund A, et al. Increased risk for cancer following sarcoidosis. Am J Respir Crit Care Med 1999; 160: 1668-1672.

10 Boffetta P, Rabkin CS, Gridley G. A cohort study of cancer among sarcoidosis patients. Int J Cancer 2009; 124: 2697-2700.

\section{Imatinib inhibits bone marrow-derived c-kit ${ }^{+}$cell mobilisation in hypoxic pulmonary hypertension}

\section{To the Editors:}

We read with great interest the review by FADINI et al. [1] in the European Respiratory Journal discussing the role of bone marrow (BM)-derived stem cells and endothelial progenitors in pulmonary hypertension $(\mathrm{PH})$. This topic is very relevant to pulmonary vascular medicine, indeed pioneering studies demonstrated that haematopoietic progenitor cells can be recruited in the pulmonary artery adventitia of neonatal animals with hypoxic $\mathrm{PH}$ [2]. In addition, BM-derived cells may express smooth muscle actin in hypoxia-remodelled pulmonary arteries, and selective depletion of circulating BM-derived precursors prevents pulmonary adventitial remodelling, suggesting that these cells may have functional relevance in the pathophysiology of hypoxia-induced PH [3]. CD117/c-kit, a transmembrane receptor tyrosine kinase for the progenitor cell factor (SCF), is a marker for BM-derived haematopoietic progenitor cells, and this receptor can be targeted by tyrosine kinase inhibitors which have been recently proposed as novel therapeutic agents to be tested in PH [4]. Indeed, a novel antiproliferative-based strategy using therapeutic agents such as imatinib mesylate inhibiting several tyrosine kinases associated with disease states, including
BCR-ABL, c-kit, and platelet-derived growth factor (PDGF) receptors $\alpha$ and $\beta$, has been demonstrated to reverse pulmonary vascular remodelling in animal models of $\mathrm{PH}$ (chronic hypoxiaand monocrotaline-induced $\mathrm{PH}$ ), through inhibition of proliferation and increased apoptosis rate of pulmonary arterial smooth muscle cells [5]. As imatinib has a broad spectrum of tyrosine kinase inhibition, we hypothesised that it may exert its beneficial effect on $\mathrm{PH}$ not only by decreasing smooth muscle cell proliferation through PDGF receptor inhibition, as initially suggested [5], but also by targeting c-kit. Hence, we wondered if administration of imatinib could attenuate experimental $\mathrm{PH}$, at least in part, by reducing the pulmonary perivascular recruitment of BM-derived c-kit ${ }^{+}$progenitor cells.

We used the murine model of $\mathrm{PH}$ induced by chronic normobaric hypoxia (10\% inspiratory oxygen fraction).

6-week old male C57BL/6J mice (Janvier, Le Genest-St-Isle, France) were exposed to $10 \%$ normobaric hypoxia for 3 weeks. Imatinib (Novartis, Horsham, UK) was orally administrated during the period of hypoxia at $10 \mathrm{mg} \cdot \mathrm{kg}^{-1} \cdot \mathrm{day}^{-1}$. Immunofluorescent staining was performed on $7-\mu \mathrm{m}$ acetone-fixed, 